\title{
The impact of diet and physical activity on skeletal muscle mass and strength in Caucasian women: implications for sarcopenia prevention
}

\author{
K. Shilland and H. D. McCarthy \\ Public Health Nutrition Research Group, London Metropolitan University, Holloway Road, London N7 8DB, UK
}

Recent research on sarcopenia suggests the need to examine the determinants of peak muscle mass attained earlier in life, with increasing attention being paid to the role of diet and physical activity in the etiology of the condition ${ }^{(1,2)}$. Such a shift in emphasis towards factors involved in the pathogenesis of sarcopenia is essential for the development of strategies aimed at reducing its prevalence. This study examined the impact of physical activity and diet on skeletal muscle mass and strength in Caucasian women and discussed the potential role of behavioural modifications in the prevention and early management of sarcopenia.

34 Caucasian women, aged between 35 and 45 years, were selected on a postcode basis in SE London. Participants completed lifestyle and physical activity questionnaires to classify them into high and low activity groups. The high activity group was further subdivided into cardiovascular only (CV), resistance only (RT) or combined CV and RT. 3 day estimated diet diaries were completed and analysed to estimate protein $(\mathrm{g} / \mathrm{kg} / \mathrm{d})$ and vitamin $\mathrm{D}(\mu \mathrm{g} / \mathrm{d})$ intakes. Height, weight and waist circumference (WC) were measured and fat mass (FM, kg), \%body fat, fat-free mass (FFM, kg) and appendicular skeletal muscle mass (SMMa) were predicted using segmental single frequency bioelectrical impedance analysis. SMMa was expressed as a percentage of total body mass (\%SMMa) and muscle-to-fat ratio (MRF) was calculated. Muscle strength was determined using a handgrip dynamometer and expressed as a ratio to body mass (GS:Mass). Independent T-tests and one-way ANOVA were used to compare anthropometric and dietary data between high and low activity groups and partial correlations, controlling for energy intake, were used to identify relationships between both diet and exercise and markers of muscle mass, strength and body composition.

SMMa $(\mathrm{kg})$ did not differ significantly between high and low activity groups $(\mathrm{p}=0.83)$, however $\%$ SMMa $(\mathrm{p}<0.01), \%$ body fat $(\mathrm{p}<0.01)$, MFR $(\mathrm{p}<0.01)$ and GS:Mass $(\mathrm{p}<0.01)$ all differed significantly between groups. Additionally, RT only and combined CV/RT groups had significantly higher \%SMMa, GS:Mass and MFR and lower \% body fat compared with the low activity group $(\mathrm{p}<0 \cdot 01)$. These measures did not differ significantly between CV only and the low activity group. SMMa (kg) was not significantly related to dietary protein $(\mathrm{g} / \mathrm{kg} / \mathrm{d})$ or vitamin $\mathrm{D}(\mu \mathrm{g} / \mathrm{d})$ intakes $(\mathrm{p}>0.05)$, whereas significant positive relationships were identified between intake of protein $(\mathrm{g} / \mathrm{kg} / \mathrm{d})$ and $\%$ SMMa $(\mathrm{p}<0.01)$, GS:Mass $(\mathrm{p}=0.01)$ and MFR $(\mathrm{p}<0 \cdot 01)$. An inverse relationship was identified between protein intake $(\mathrm{g} / \mathrm{kg} / \mathrm{d})$ and $\%$ body fat $(\mathrm{p}=0.01)$. Vitamin $\mathrm{D}(\mu \mathrm{g} / \mathrm{d})$ intake was positively correlated with GS: Mass $(p=0.01)$ in the high activity group alone and participants taking vitamin D supplements had significantly greater $\%$ SMMa $(p<0 \cdot 01)$, GS:Mass $(p=0 \cdot 01)$ and MFR ratio $(p=0 \cdot 01)$ than non-consumers.

These findings indicate that dietary intakes of protein $(\mathrm{g} / \mathrm{kg} / \mathrm{d})$ and vitamin $\mathrm{D}(\mu \mathrm{g} / \mathrm{d})$, together with physical activity, in particular $\mathrm{RT}$, could play a key role in the prevention or delay of sarcopenia, mediated through their positive influence on body composition rather than muscle mass alone. Since diet and physical activity are both modifiable factors, these findings suggest that guidelines that promote resistance exercise and highlight the importance of dietary protein and vitamin D across the life-course may have the potential to attenuate the risk of sarcopenia and improve long-term health and economic outcomes for today's ageing population. However, further research is required to substantiate these recommendations.

1. Sayer AA, Robinson SM, Patel HP et al. (2013) Age Ageing 42, 145-50.

2. Robinson S, Cooper C, Aihie Sayer A. (2012) J Aging Res 2012:510801. Epub 2012 Mar 15. 